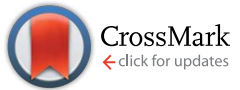

Cite this: Chem. Sci., 2014, 5, 4794

\title{
Chromo-pharmacophores: photochromic diarylmaleimide inhibitors for sirtuins $\uparrow$
}

\author{
C. Falenczyk, ${ }^{a}$ M. Schiedel, ${ }^{b}$ B. Karaman, ${ }^{c}$ T. Rumpf, $^{b}$ N. Kuzmanovic, ${ }^{a}$ M. Grøtli, ${ }^{d}$ \\ W. Sippl, ${ }^{c}$ M. Jung ${ }^{b}$ and B. König*a
}

Controlling the activity of sirtuins is of high biomedical relevance as the enzymes are involved in cancer, neurodegeneration and other diseases. Therefore structural elements of 3,4-bisindoylmaleimides (BIMs), which are known $\mathrm{NAD}^{+}$-dependent histone deacetylase (sirtuin) inhibitors, were merged with photochromic diarylmaleimides to yield photoswitchable enzyme inhibitors. The new inhibitors show excellent photophysical properties, are switchable even in polar solvents, and subtype selective against hSirt2. The inhibitory activity changes up to a factor of 22 for the two photoisomers and physiological properties can therefore be effectively toggled by irradiation with light of different wavelengths. Docking experiments using the enzyme crystal structure explain the observed activity changes based on the steric demand of the thiophene substitution and the rigidity of the molecular structure.

Received 8th May 2014

Accepted 6th August 2014

DOI: $10.1039 / \mathrm{c} 4 \mathrm{sc} 01346 \mathrm{~h}$

www.rsc.org/chemicalscience

typically a high switching fatigue resistance. In comparison to

\section{Introduction}

Photochromism has recently received increasing attention due to a variety of potential applications in molecular optoelectronics, optical data storage and as molecular switches..$^{1-3}$ The interest in photopharmacology is revealed by the increasing number of reported photochromic enzyme inhibitors, ${ }^{4-6}$ ion channel blockers ${ }^{7}$ and receptor ligands ${ }^{8,9}$ demonstrating the importance of such compounds as tools for the investigation of cellular processes and pathogeneses in life sciences. ${ }^{10}$ Two strategies are applied for controlling enzyme activity by light: the photochromic moiety can either be attached to the scaffold of a known substrate or is the linker between two ligands. The latter approach allows the light-induced change of the ligand spacing, which is useful for targets with two binding sites. ${ }^{2}$ Various types of photochromic compounds have been developed so far, for instance spiropyranes, azobenzenes, fulgides and diarylethenes (DTEs). ${ }^{2}$ Diarylmaleimide derivatives are well known for their photochromic properties and their ability to change conformational mobility reversibly upon irradiation (Fig. 1). Both photoisomers are thermally stable and show

${ }^{a}$ Institute of Organic Chemistry, University of Regensburg, 93040 Regensburg, Germany. E-mail: burkhard.koenig@chemie.uni-regensburg.de

${ }^{b}$ Institute of Pharmaceutical Sciences, University of Freiburg, 79104 Freiburg, Germany 'Department of Pharmaceutical Chemistry, Martin Luther University of Halle Wittenberg, 06120 Halle/Saale, Germany

${ }^{d}$ Department of Chemistry and Molecular Biology, University of Gothenburg, 41296 Göteborg, Sweden

$\dagger$ Electronic supplementary information (ESI) available: Detailed experimental procedures of the synthesis of $\mathbf{3}$ and 11, additional spectral data, photochemical measurements, biological data and docking studies of 2, 3 and 11. See DOI: $10.1039 / \mathrm{c} 4 \mathrm{sc} 01346 \mathrm{~h}$ other DTEs, the absorption of diarylmaleimides is shifted bathochromically and therefore the isomerization is possible at longer wavelengths, which reduces cell damage, otherwise caused by high energy light. The optical properties and the slightly better water solubility makes them interesting candidates for photochromic inhibitors. ${ }^{1}$ However, to be useful in biological applications, photochromic compounds should also undergo photochromic reactions in polar solvents. One handicap of diarylmaleimides is that these reactions are disfavoured in polar solvents due to a twisted intramolecular electron charge transfer (TICT) from the donor aryl part to the electron acceptor maleimide moiety. ${ }^{11-13}$

There is an ongoing interest in the regulation of enzyme activity in the field of epigenetics, particularly with regard to anticancer research. $\mathrm{NAD}^{+}$-dependent histone deacetylases also termed sirtuins are enzymes, which deacetylate histones and certain non-histone proteins. Sirtuins (Sirt1-7) represent the class III of four histone deacetylases (HDACs). Their activity is

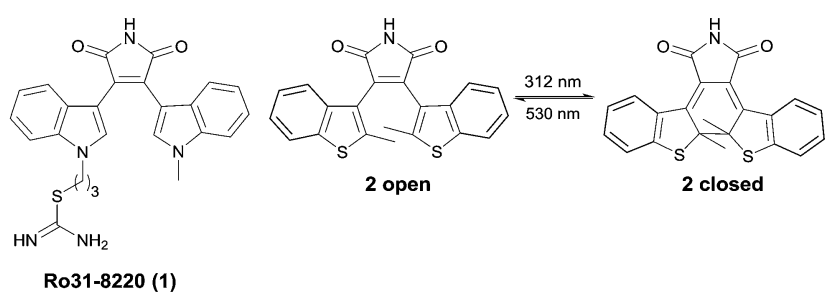

Fig. 1 Sirtuin inhibitor 1 (Ro31-8220) and by way of example the structural related photochromic diarylmaleimide 2 , which interconvert reversible by irradiation with light of different wavelength between the open and closed photoisomer. 
dependent on nicotinamide adenine dinucleotide $\left(\mathrm{NAD}^{+}\right)$, whereas the other classes are zinc-dependent amidohydrolases. ${ }^{\mathbf{1 4 - 1 6}}$ The biological activity of sirtuins has been linked to the pathogenesis of cancer $^{17}$ as well as viral, ${ }^{18}$ metabolic ${ }^{19,20}$ and neurological diseases. ${ }^{21,22}$ As several disorders are linked to the perturbation of HDACs, they are a promising target for drug discovery. Various class I and II HDACs anticancer drugs are already in clinical trials or approved as therapeutics. ${ }^{23}$ To get more insight into the biological activity and molecular mechanism of all class III HDAC members there is a need to investigate potent and isotype selective small molecule modulators for human sirtuins. Several lead structures for small-molecule sirtuin inhibitors have been identified so far. ${ }^{16}$ Besides nicotinamide as the endogenous inhibitor, splitomicins, suramins, indoles as well as kinase inhibitors like bisindoylmaleimides (BIMs) are of particular interest. ${ }^{\mathbf{1 4 - 1 6}}$ Diarylmaleimides are stable photochromic compounds and structurally related to BIMs like 1 (Ro31-8220), a potent sirtuin inhibitor (Fig. 1). ${ }^{14} \mathrm{We}$ envisioned that merging the structure of the DTE chromophore and the BIM pharmacophore to new diarylmaleimides would lead to photochromic sirtuin inhibitors. Variation of the substitution pattern and molecular docking experiments provides insight into the binding mode of the diarylmaleimides and their photoisomers to the active site of the enzyme and thereby lead to potent and isotype selective inhibitors for sirtuins.

\section{Results and discussion}

\section{Synthesis}

A common strategy for the synthesis of 3,4-diarylmaleimides is the intramolecular Perkin-type condensation. ${ }^{24,25}$ The synthesis of compound 2 (Fig. 2) was previously described by Scandola et $a l^{3}$ Adapted from this synthesis, the non-symmetric maleimide 3 was prepared in a similar manner with further functionalization by Suzuki coupling (see ESI $\dagger$ ).

Unfortunately, compound $\mathbf{1 1}$ could not be obtained by this strategy. Hence, we developed an alternative route for the synthesis of 11, which is outlined in Scheme 1. Starting from 5chloro-2-methyl-thiophene (4) standard Friedel-Crafts conditions yielded ketone 5 and glyoxylate ester 8 . The ester $\mathbf{6}$ was obtained via an oxidative rearrangement from ketone 5. Suzuki coupling yielded the functionalized esters 7 and 9. Compound $\mathbf{1 0}$ was prepared by aminolysis of glyoxylate ester 9, which was subsequently used in a Perkin condensation with ester 7 to obtain maleimide 11. Scheme 1 summarizes the synthesis of the symmetric diarylmaleimide 11.

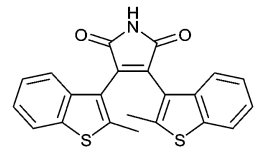

2

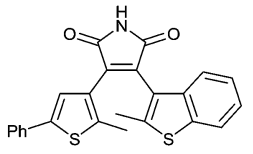

3
Fig. 2 Structures of diarylmaleimides 2 and 3.

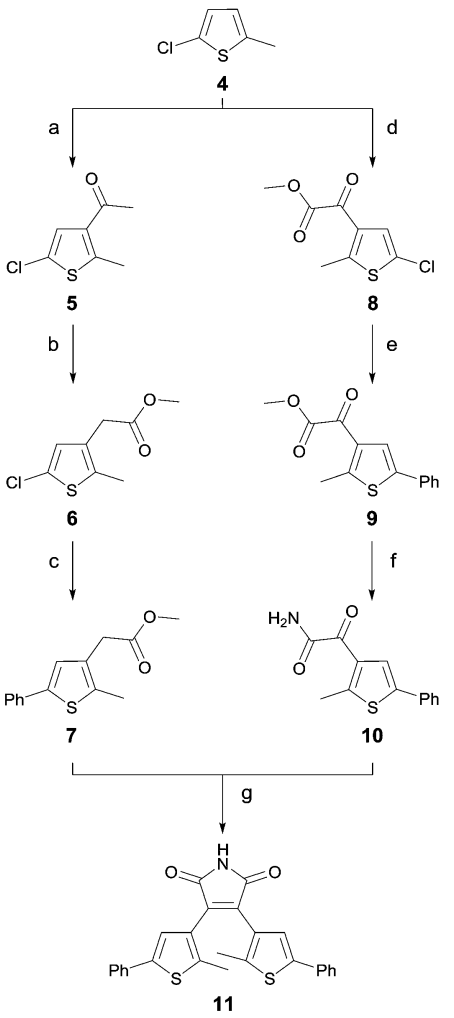

Scheme 1 Synthetic strategy with reagents and conditions for 11: (a) acetyl chloride, $\mathrm{AlCl}_{3}, \mathrm{CH}_{2} \mathrm{Cl}_{2}, 0^{\circ} \mathrm{C}, 2 \mathrm{~h}$, (84\%); (b) TTN, $\mathrm{HClO}_{4}, \mathrm{MeOH}$, $\mathrm{RT}$, overnight, (83\%); (c) $\mathrm{Pd}_{2}(\mathrm{dba})_{3}, \mathrm{XPhos}$, phenylboronic acid, $\mathrm{K}_{3} \mathrm{PO}_{4}$, 1,4-dioxane, $100^{\circ} \mathrm{C}$, overnight, (52\%); (d) methyl chlorooxoacetate, $\mathrm{AlCl}_{3}, \mathrm{CH}_{2} \mathrm{Cl}_{2}, 0^{\circ} \mathrm{C}, 2 \mathrm{~h}$, (73\%); (e) $\mathrm{Pd}_{2}(\mathrm{dba})_{3}, \mathrm{XPhos}$, phenylboronic acid, $\mathrm{K}_{3} \mathrm{PO}_{4}, 1,4$-dioxane, $100{ }^{\circ} \mathrm{C}$, overnight, (63\%); (f) aq. $\mathrm{NH}_{3}, \mathrm{THF}, \mathrm{RT}$, overnight, (80\%); and (g) KOtBu (1 M in THF), THF, $0{ }^{\circ} \mathrm{C} \rightarrow \mathrm{RT}$, overnight, (17\%).

\section{Photochromic properties}

The photoisomerization of photochromic compounds in polar solvents is essential for applications in biological assays. As dimethyl sulfoxide is a standard solvent for inhibitor screening, this solvent was used to investigate the switching ability of the diarylmaleimides. The ring closure is suppressed in polar solvents due to a twisted intramolecular electron transfer. This phenomenon is well-investigated for maleimides bearing two benzothiophenes. ${ }^{12}$ To enhance the switching ability of DTEs the introduction of sterical hindrance either by bulky substituents on the aryl moieties or on the bridge is a widely applied concept. ${ }^{26-28}$ As seen from the perspective of the pharmacophore only the introduction of sterically demanding substituents on the aryl moieties are possible. Otherwise the ability to form relevant hydrogen bonds between the maleimide and the backbone of the enzyme is lost. We decided to replace the benzothiophene moieties by phenyl substituted thiophenes. Despite this change the structure of the chromophore is still similar to the pharmacophore, but the photochromic properties improve significant. The exchange of the methyl-benzothiophenes in 2 by one or two methyl-phenyl substituted thiophenes in 3 or 11, resp., stabilizes the antiparallel conformation, which favors the photochemical ring closure reaction. 


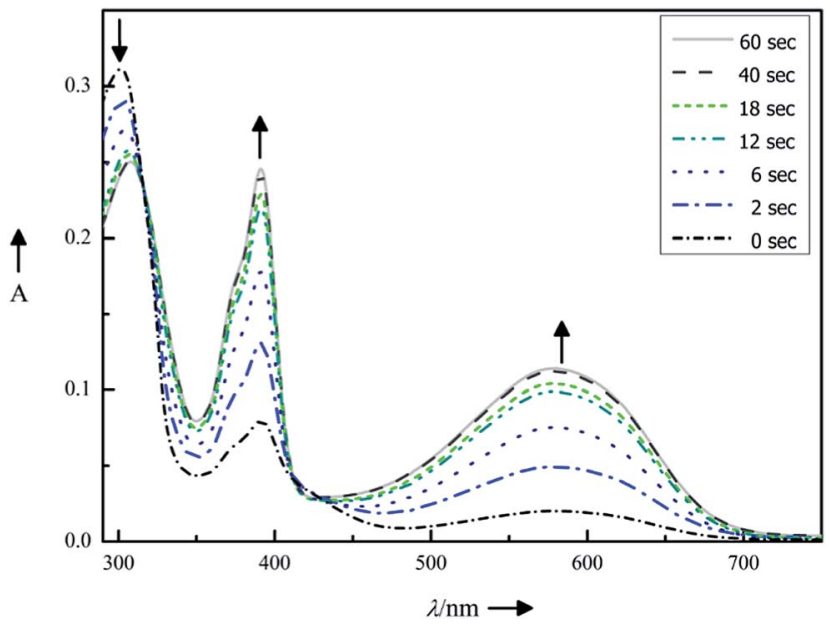

Fig. 3 Photochromic properties of compound 11 (10 $\mu \mathrm{m}$ in DMSO): absorption spectra changes upon continuous irradiation for $0,2,6,12$, 18,40 and $60 \mathrm{~s}$ with light of $\lambda_{\max }=312 \mathrm{~nm}$.

The photoisomerization was monitored by absorption spectroscopy (Fig. 3) and the photostationary states (PSS) were determined by HPLC measurements (see ESI $\dagger$ ).

Upon irradiating $(\lambda=312 \mathrm{~nm})$ the dissolved compounds 2,3 and 11, a rapid color change was observed, resulting in new absorption bands characteristic for each compound. The photochromic properties are summarized in Table 1. Replacing the benzothiophene moieties by phenyl-substituted thiophenes has two consequences: (1) the absorbance maxima for the closed isomers shift bathochromically from compound 2 to compound 11. Therefore light of longer wavelength is sufficient for the isomerization. (2) The photoconversion becomes more efficient as indicated by PSS increasing from $62 \%$ to $94 \%$. Such high PSS is advantageous for binding investigations as the interaction with the enzyme can be restricted to mainly one photoisomer.

By way of example, Fig. 3 illustrates the photoisomerization of compound 11. Upon irradiating $(\lambda=312 \mathrm{~nm})$ of compound 11 in dimethyl sulfoxide solution, a rapid color change from light green to deep purple was observed, resulting in new absorption bands at $390 \mathrm{~nm}$ and $580 \mathrm{~nm}$ (marked with black arrows in Fig. 3). The isosbestic points (Table 1) indicate a clean two-component switching. Repetition of the photoisomerization of compound $\mathbf{1 1}$ shows a good fatigue resistance over five cycles (see ESI†).

Table 1 Photochromic properties of diarylmaleimides 2, 3 and 11 (10 $\mu \mathrm{m}$ in DMSO)

\begin{tabular}{llllll}
\hline & & $\begin{array}{l}\lambda_{\max } \text { open } \\
{[\mathrm{nm}]}\end{array}$ & $\begin{array}{l}\lambda_{\max } \text { closed } \\
{[\mathrm{nm}]}\end{array}$ & $\begin{array}{l}\text { Isosbestic } \\
\text { points }[\mathrm{nm}]\end{array}$ & PSS $^{a}$ \\
\hline 1 & $\mathbf{2}$ & 278 & 353,495 & 293 & $62 \%$ \\
2 & $\mathbf{3}$ & 274 & 329,527 & 291 & $87 \%$ \\
3 & $\mathbf{1 1}$ & 290 & 390,580 & $316,410,430$ & $94 \%$
\end{tabular}

${ }^{a}$ Determined by HPLC measurements.

\section{Enzyme inhibition}

Next, the inhibitory effect of the photoresponsive compounds 2 , $\mathbf{3}$ and $\mathbf{1 1}$ against sirtuins in a homogenous fluorescent assay, previously established by Jung et al. was determined. The enzymatic conversion was quantified using $(S)$-[5-acetylamino1-(4-methyl-2-oxo-2 $H$-chromen-7-ylcarbamoyl)-pentyl]-carbamic acid benzyl ester also termed $\mathrm{ZMAL}^{29}$ as fluorescent substrate for deacetylation followed by a tryptic digestion step. ${ }^{30} \mathbf{1}$ (Ro318220) has an $\mathrm{IC}_{50}$ value of $0.8 \mu \mathrm{m}$ and is the most potent BIM inhibitor reported so far for human Sirt2 (hSirt2). ${ }^{\mathbf{1 4 , 1 6}}$ In comparison to $\mathbf{1}$ the photoresponsive diarylmaleimides $\mathbf{3}$ and 11 showed similar inhibition strength against hSirt2. The respective $\mathrm{IC}_{50}$ values are given in Table 2 .

The scaffold of compound 2 is structurally closest related to 1. Its open isomer exhibits a lower $\mathrm{IC}_{50}$ value against hSirt2 (Table 2, entry 6) than its closed photoisomer. By the exchange of one of the benzothiophenes in 2 by a phenyl substituted thiophene as in compound $\mathbf{3}$ the inhibition activity increases. However, the open and the closed photoisomer of compound 3 show the same inhibition (Table 2, entries 4 and 5). The replacement of the second benzothiophene from the maleimide moiety by a phenyl substituted thiophene in compound $\mathbf{1 1}$ yields an inhibition corresponding to an $\mathrm{IC}_{50}$ value of $4.2 \mu \mathrm{m}$ of the open photoisomer. The ring closed photoisomer of $\mathbf{1 1}$ shows a twentyfold lower enzyme inhibition with $92.3 \mu \mathrm{m}$ (Table 2, entries 2 and 3). Diarylmaleimide 11 gives the most significant change in hSirt2 inhibition activity for both photoisomers and therefore its isotype selectivity against human Sirt1 (hSirt1) and human Sirt3 (hSirt3) under the same assay conditions $^{\mathbf{3 0}}$ as used for hSirt2 were determined. The isotype selectivity for BIM 1 (Ro31-8220) is only small (Table 2, entry 1). Therefore inhibitor concentrations at $20 \mu \mathrm{m}$ and $200 \mu \mathrm{m}$ were used to determine the enzyme activity using nicotinamide as a reference. Neither the open isomer nor the closed isomer of $\mathbf{1 1}$ inhibited hSirt1 or hSirt3 significantly (Table 2, entries 2 and 3).

Next, the enzyme activity of hSirt 2 was controlled by light under assay conditions. Therefore, the photochemical conversion from the closed isomer to the open isomer of $\mathbf{1 1}$ and the corresponding inhibitory activity were measured as a function of time (Fig. 4). Hence the less potent closed isomer of 11 was incubated with the enzyme under assay conditions and irradiated over a period of $30 \mathrm{~min}$ with a green LED $(2.5 \mathrm{~W}, 530 \mathrm{~nm}$ emission maximum). Then the cofactor $\mathrm{NAD}^{+}$and $\mathrm{ZMAL}$ were added and the assay was performed under the usual conditions. Fig. 4 illustrates the time course of the isomerization (dotted line) and inhibitory activity (straight line) of 11. The irradiated samples switched from the less potent closed form of $\mathbf{1 1}$ to the significantly more potent open form.

After 20 min complete photochemical conversion of $\mathbf{1 1}$ and a constant inhibition value are observed. The determined values correspond to the inhibition activity of the open isomer of $\mathbf{1 1}$ (Table 2) proving that the photoisomerization is possible in the presence of the enzyme under assay conditions.

Additionally, the open photoisomer of $\mathbf{1 1}$ was tested for cellular activity using hyperacetylation of tubulin ${ }^{31}$ as a marker. 11 showed tubulin hyperacetylation and hence target 
Table $2 I C_{50}$ values of the photochromic diarylmaleimides 2, 3 and 11 and 1 (Ro31-8220) as BIM reference

\begin{tabular}{|c|c|c|c|c|c|}
\hline Entry & Compound & hSirt1 $\mathrm{IC}_{50}[\mu \mathrm{M}]$ & hSirt2 $\mathrm{IC}_{50}[\mu \mathrm{M}]$ & hSirt3 $\mathrm{IC}_{50}[\mu \mathrm{M}]$ & hSirt2 $\mathrm{IC}_{50}$ closed $/ \mathrm{IC}_{50}$ open \\
\hline 1 & 1 (ref. 14 and 15) & $3.5 \pm 0.4$ & $0.8 \pm 0.2$ & $3.7 \pm 0.2$ & \\
\hline 2 & 11 open & n.i. ${ }^{a}$ & $4.2 \pm 0.8$ & n.i. & 22 \\
\hline 4 & 3 open & $-^{b}$ & $2.3 \pm 0.4$ & - & 1 \\
\hline 5 & 3 closed & - & $2.1 \pm 0.3$ & - & \\
\hline 6 & 2 open & - & $12.9 \pm 5.4$ & - & 2 \\
\hline
\end{tabular}

engagement similar to the control inhibitor 1 (Ro31-8220) ${ }^{\mathbf{1 4}}$ (see ESI $\dagger$ ). But further studies are required as we cannot rule out indirect effects as the compounds have not been optimized for selectivity, e.g. against kinases.

\section{Molecular docking}

In order to rationalize the observed in vitro activities docking of the open and closed form of the diarylmaleimides to hSirt2 (PDB-ID 3ZGO) was carried out. Docking of the open form of the isomers 2, $\mathbf{3}$ and $\mathbf{1 1}$ to hSirt2 resulted in a similar interaction at the acetyl-lysine channel of hSirt 2 (see ESI $\dagger$ ) favoured by a hydrogen bond with the backbone of Val233 and van-der-Waals interactions with hydrophobic and aromatic residues (Phe96, Phe119, Ile169, His187, Phe234, and Phe235). Val233 represents a conserved amino acid in sirtuins that binds the amide group of the acetyl-lysine substrate. The open photoisomers fit nicely to the acetyl-lysine pocket of hSirt2. The angle between the aromatic rings and the maleimide is for all open photoisomers around $-50 \mathrm{deg}$., which allows the accommodation of both aromatic substituents in the acetyl-lysine pocket. The closed photoisomer of $\mathbf{1 1}$ might be too big (distance from both ends of the phenyl ring $14.7 \AA$ compared to $12.9 \AA$ for 3 and $10.9 \AA$ for 2 ) and rigid to interact in a similar way within the binding pocket as the open and flexible isomer of $\mathbf{1 1}$ (Fig. 5). The docking

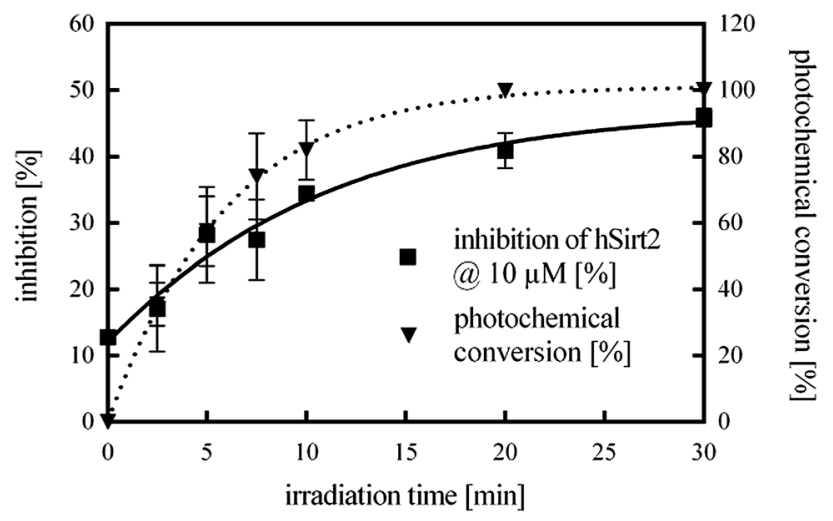

Fig. 4 Time course of the photochemical conversion from the closed to the open form of 11 (dotted line) and the corresponding inhibitory activity against hSirt2 (straight line) upon continuous irradiation with light of $\lambda_{\max }=530 \mathrm{~nm}$ for $0,2.5,5,7.5,10,20$ and 30 min at an assay concentration of $10 \mu \mathrm{M}$. results suggest that the terminal aromatic rings and methyl groups clash with His187 and Phe235 resulting in a different orientation at the binding pocket compared to the closed isomers of 2 and 3 (Fig. 6). As a consequence, the distance between the maleimide and Val233 backbone carbonyl group is increased (3.85 $\mathrm{A}$ ) disabling the hydrogen bond formation (see ESI $\dagger$ ). Only the closed photoisomers of 2 and 3 fit well to the binding pocket and make favourable van-der-Waals interactions with the aromatic and hydrophobic residues (see ESI $\dagger$ ).

Docking to the apo Sirt3 (PDB-ID 3GLS) substrate binding pocket was not possible due to a different conformation of the flexible loop flanking the acetyl-lysine channel. Especially, the residues Phe157, Arg158 and Phe294 block part of the substrate binding pocket observed in hSirt2 (see ESI $\dagger$ ). Thus, the docking suggests that the observed selectivity of the photochromic inhibitor $\mathbf{1 1}$ arises from a different orientation of amino acid residues located at the entrance of the acetyl-lysine channel of hSirt3.

In case of apo hSirt1 (PDB-ID 4IG9), which shows also an open conformation of the substrate binding site, the docking of the photoisomers of $\mathbf{1 1}$ resulted in less favourable complexes.

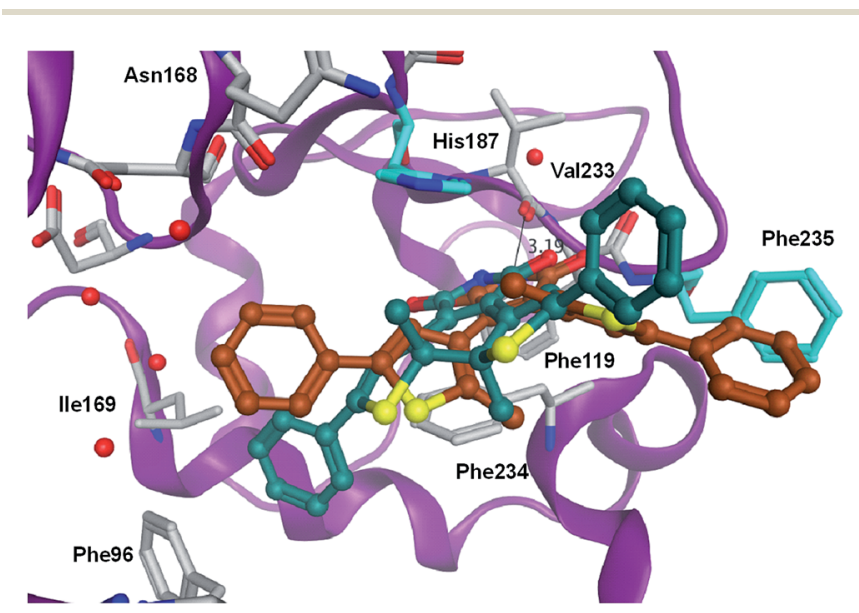

Fig. 5 Comparison of the binding mode of photoisomer 11 at hSirt2 in the open (brown) and the closed form (dark green). The two residues (Phe235 and His187) that hinder the proper orientation of the closed photoisomer 11 are colored cyan. Water molecules are shown as red spheres. The hydrogen bond to the backbone of Val233 is shown as black line (distance between the $\mathrm{NH}$ group of closed 11 and the backbone carbonyl of Val233 is $3.19 \AA$ ). 


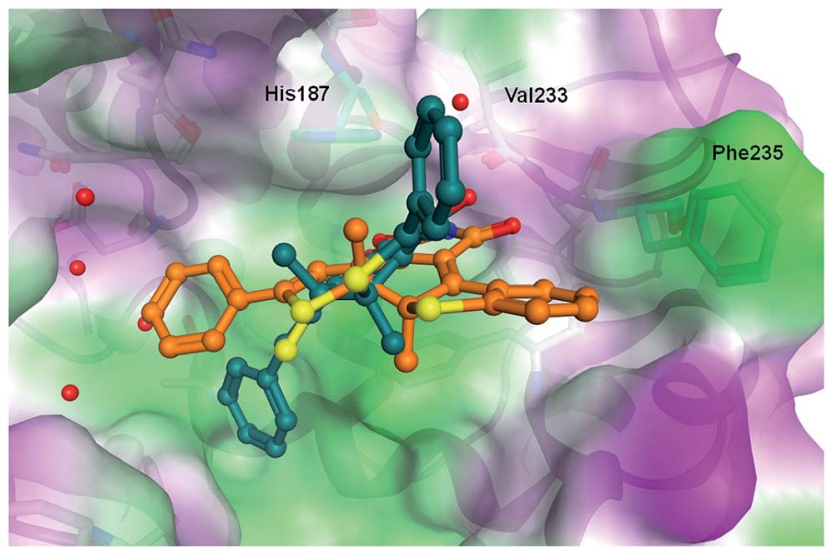

Fig. 6 Comparison of the binding mode of closed photoisomer 3 (orange) and closed photoisomer 11 (dark green) at hSirt2. Water molecules are shown as red spheres.

Due to the orientation of Phe414 the pocket shows a different shape compared to hSirt2 which hinders the formation of a hydrogen bond between Val233 and the maleimide group of the docked inhibitors (see ESI $\dagger$ ). Compound 11 is therefore isotype selective for hSirt2 and although 11 shows no higher potency than $\mathbf{1}$, it is the first example for a potent photochromic maleimide sirtuin inhibitor with such selectivity and the first example for a photochromic inhibitor combining elements of a chromophore and a pharmacophore within one structure.

\section{Conclusions}

In summary, we have merged structural elements of the well investigated photochromic diarylmaleimides with biological relevant bisindoylmaleimides, which are known for their sirtuin inhibitory activity, yielding potent and isotype selective photochromic inhibitors. The new compounds are the first photochromic epigenetic inhibitors described so far. The photochromic and enzyme inhibition properties of the new diarylmaleimides were optimized by variation of the maleimide scaffold. Exchange of the benzothiophene moieties by phenyl substituted thiophenes led to nearly quantitative photostationary states of the diarylmaleimides in dimethyl sulfoxide and subtype selective inhibition of hSirt2 in the low micromolar range. The photoisomers of diarylmaleimide $\mathbf{1 1}$ differ more than 20-fold in their inhibition ability. Irradiation of the closed photoisomer of $\mathbf{1 1}$ in the presence of the enzyme isomerizes the compound into the more potent open photoisomer allowing the remote control of the enzyme activity by green light. The inhibitory activity of the open photoisomer of $\mathbf{1 1}$ was also tested in cell culture. 11 induced hyperacetylation of tubulin, but further studies are necessary to eliminate indirect effects.

The new diarylmaleimides combine in their molecular structure photochromic and selective enzyme inhibiting properties. They are valuable tools for the investigation of molecular binding mechanisms of sirtuins and the application of the concept to other privileged structures of the bisindoylmaleimide family may be readily envisaged.

\section{Acknowledgements}

Financial support by the Deutsche Forschungsgemeinschaft (DFG) within the GRK 1910 and within the CRC992 (MEDEP, Medical Epigenetics) is gratefully acknowledged. We thank Karin Schmidtkunz for western blot experiments.

\section{Notes and references}

1 M. Irie, Chem. Rev., 2000, 100, 1685-1716.

2 W. Szymański, J. M. Beierle, H. A. V. Kistemaker, W. A. Velema and B. L. Feringa, Chem. Rev., 2013, 113, 6114-6178.

3 M. T. Indelli, S. Carli, M. Ghirotti, C. Chiorboli, M. Ravaglia, M. Garavelli and F. Scandola, J. Am. Chem. Soc., 2008, 130, 7286-7299.

4 D. Vomasta, A. Innocenti, B. König and C. T. Supuran, Bioorg. Med. Chem. Lett., 2009, 19, 1283-1286.

5 D. Vomasta, C. Högner, N. R. Branda and B. König, Angew. Chem., Int. Ed., 2008, 47, 7644-7647.

6 B. Reisinger, N. Kuzmanovic, P. Löffler, R. Merkl, B. König and R. Sterner, Angew. Chem., Int. Ed., 2014, 53, 595-598.

7 M. R. Banghart, A. Mourot, D. L. Fortin, J. Z. Yao, R. H. Kramer and D. Trauner, Angew. Chem., Int. Ed., 2009, 48, 9097-9101.

8 M. Volgraf, P. Gorostiza, S. Szobota, M. R. Helix, E. Y. Isacoff and D. Trauner, J. Am. Chem. Soc., 2006, 129, 260-261.

9 M. Schönberger and D. Trauner, Angew. Chem. Int. Ed., 2014, 53, 3264-3267.

10 W. A. Velema, W. Szymanski and B. L. Feringa, J. Am. Chem. Soc., 2014, 136, 2178-2191.

11 T. Yamaguchi and M. Irie, Chem. Lett., 2004, 33, 1398-1399.

12 T. Yamaguchi, K. Uchida and M. Irie, J. Am. Chem. Soc., 1997, 119, 6066-6071.

13 M. Irie and K. Sayo, J. Phys. Chem., 1992, 96, 7671-7674.

14 J. Trapp, A. Jochum, R. Meier, L. Saunders, B. Marshall, C. Kunick, E. Verdin, P. Goekjian, W. Sippl and M. Jung, J. Med. Chem., 2006, 49, 7307-7316.

15 M. Freitag, J. Schemies, T. Larsen, K. El Gaghlab, F. Schulz, T. Rumpf, M. Jung and A. Link, Bioorg. Med. Chem., 2011, 19, 3669-3677.

16 M. Lawson, U. Uciechowska, J. Schemies, T. Rumpf, M. Jung and W. Sippl, Biochim. Biophys. Acta, Gene Regul. Mech., 2010, 1799, 726-739.

17 B. Peck, C.-Y. Chen, K.-K. Ho, P. Di Fruscia, S. S. Myatt, R. C. Coombes, M. J. Fuchter, C.-D. Hsiao and E. W.-F. Lam, Mol. Cancer Ther., 2010, 9, 844-855.

18 M. R. Pinzone, B. Cacopardo, F. Condorelli, M. Di Rosa and G. Nunnari, Curr. Drug Targets, 2013, 14, 648-652.

19 G. Leonard, Nature, 2006, 444, 868-874.

20 H. H. Riekelt, P. Eija and A. Johan, Nat. Rev. Mol. Cell Biol., 2012, 13, 225-238.

21 T. F. Outeiro, E. Kontopoulos, S. M. Altmann, I. Kufareva, K. E. Strathearn, A. M. Amore, C. B. Volk, M. M. Maxwell, J.-C. Rochet, P. J. McLean, A. B. Young, R. Abagyan, M. B. Feany, B. T. Hyman and A. G. Kazantsev, Science, 2007, 317, 516-519. 
22 G. Jun, W. Wen-Yuan, M. Ying-Wei, G. Johannes, G. Ji-Song, P. Ling, M. Gloria, K. Dohoon, C. S. Susan and T. Li-Huei, Nature, 2010, 466, 1105-1109.

23 M. Slingerland, H.-J. Guchelaar and H. Gelderblom, AntiCancer Drugs, 2014, 25, 140-149.

24 M. M. Faul, L. L. Winneroski and C. A. Krumrich, J. Org. Chem., 1998, 63, 6053-6058.

25 M. M. Faul, L. L. Winneroski and C. A. Krumrich, Tetrahedron Lett., 1999, 40, 1109-1112.

26 R. Göstl, B. Kobin, L. Grubert, M. Pätzel and S. Hecht, Chem. -Eur. J., 2012, 18, 14282-14285.
27 W. Li, C. Jiao, X. Li, Y. Xie, K. Nakatani, H. Tian and W. Zhu, Angew. Chem., Int. Ed., 2014, 53, 4603-4607.

28 C. Elsner, T. Cordes, P. Dietrich, M. Zastrow, T. T. Herzog, K. Rück-Braun and W. Zinth, J. Phys. Chem. A, 2009, 113, 1033-1039.

29 B. Heltweg, F. Dequiedt, E. Verdin and M. Jung, Anal. Biochem., 2003, 319, 42-48.

30 B. Heltweg, J. Trapp and M. Jung, Methods, 2005, 36, 332337.

31 B. J. North, B. L. Marshall, M. T. Borra, J. M. Denu and E. Verdin, Mol. Cell, 2003, 11, 437-444. 